\title{
Study of Heavily Adhesive Cs in Soil Environment
}

\author{
K. Sato and K. Numata
}

\begin{abstract}
Two years having passed since the nuclear crisis at Fukushima, radioactive ${ }^{137} \mathrm{Cs}$ with the half-life of $\sim 30$ years has come to the forefront of our largest concern. To gain an insight into current unsuccessful ${ }^{137} \mathrm{Cs}$ decontamination from soil, heavily adhesive Cs adsorption, referred as specific Cs adsorption here, is highlighted for the layered clay minerals. Beside the interlayer $\mathrm{Cs}^{+}$cations, a population of $\mathrm{Cs}$ is able to adsorb on the surfaces of open nanospaces with their sizes of $\sim$ $0.3 \mathrm{~nm}$ and $\sim 0.9 \mathrm{~nm}$, which are formed by one- and two-clay nanosheet insertion into interlayer spaces. They are adsorbed on the surfaces of both the open nanospaces so strongly that cannot be removed even by the hydrochloric acid solution of $\mathrm{pH}$ 1.0, these open nanospaces thus acting as the specific Cs adsorption site. The characteristic local molecular structures as a clay-nanosheet edge and a wedge-shaped frayed part available in the open nanospaces are responsible for the specific Cs adsorption. Radioactive ${ }^{137} \mathrm{Cs}$ that is not cleaned up after the decontamination work would originate from the specific Cs adsorption clarified here. The present findings unambiguously provide the molecular evidence of specific Cs adsorption and are thus of importance for further sophisticated decontamination of the radioactive Cs from the soil environment.
\end{abstract}

Index Terms-Clay mineral, positronium, radioactive Cs, soil environment.

\section{INTRODUCTION}

Following the nuclear crisis at the Fukushima Daiichi Nuclear Power Plant triggered by the earthquake off the Pacific coast of Tohoku, Japan, on March 11, 2011 [1], a number of volatile fission products as, e.g., ${ }^{129 \mathrm{~m}} \mathrm{Te},{ }^{131} \mathrm{I},{ }^{134} \mathrm{Cs}$, ${ }^{136} \mathrm{Cs}$, and ${ }^{137} \mathrm{Cs}$ have been released into the atmosphere [2]. These radioactive nuclides were carried by wind and fell out on the land surface, by which soil contamination occurs [3]-[5]. Two years having passed, the radionuclide of our largest concern is ${ }^{137} \mathrm{Cs}$ with the half-life of $\sim 30$ years because of its harmful influences on human life for decades [6], [7]. The radioactive Cs deposited on the terrestrial areas is estimated to be $\sim 22 \%$ of the total release that largely remains in soil [8].

In the soil environment, there exists a wealth of layered clay minerals such as saponite. The layered clay minerals with angstrom-scale interlayer spaces act as the major sorbents of radioactive Cs from the following reasons [9]. The layers of clay minerals are negatively charged originating from local compositional deviation, which is generally balanced with alkali cations, such as $\mathrm{Na}^{+}, \mathrm{Li}^{+}$, and

Manuscript received July 8, 2013; revised August 20, 2013. This work was partially supported by a Grant-in-Aid of the Japanese Ministry of Education, Science, Sports and Culture (Grant Nos. 25400318 and 25400319).

The authors are with Department of Environmental Sciences, Tokyo Gakugei University, 4-1-1 Koganei, Tokyo 184-8501, Japan (e-mail: sato-k@u-gakugei.ac.jp; m121817m@st.u-gakugei.ac.jp).
$\mathrm{Cs}^{+}$in the interlayer spaces (see Fig. 1). The $\mathrm{Cs}^{+}$cation has a radius of $\sim 1.8 \AA$ larger than those of other alkali cations, being less hydrated with water molecules to go outside the interlayer spaces. Furthermore, there exists a hexagonal cavity with the size of $\sim 2.6 \AA$ on the layer surface, which is suitable for capturing the $\mathrm{Cs}^{+}$cation. It is thus normally accepted that the layer surface of clay minerals is adsorption sites for radioactive Cs.

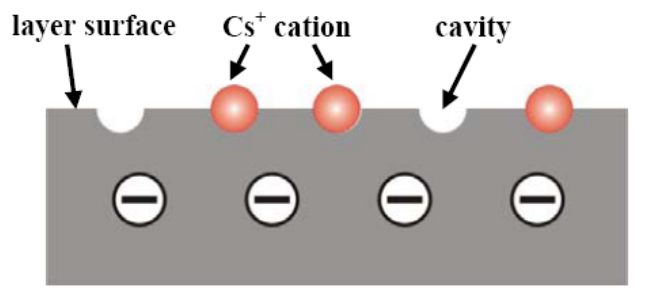

Fig. 1. Schematic illustration of $\mathrm{Cs}+$ adsorption on the layer surface of clay mineral.

Off-site decontamination of radioactive nuclides has been extensively attempted by municipals, such as Fukushima city, under the instruction of the Ministry of the Environment (MOE), but there seems to be little progress in the radioactive Cs [10]. According to the results of radiation monitoring survey by the Ministry of Education, Culture, Sports, Science and Technology (MEXT), many hot spots as for the radioactive Cs exceeding the provisory reference value still remain inside and outside Fukushima [11]. It has been reported that the decontamination works performed as the model project of clean-up methodology drawn up by MOE and Japan Atomic Energy Agency (JAEA) significantly reduces the dose rate but does not achieve the safety level down to the annual dose of $\sim 20 \mathrm{mSv}$ [12]. Indeed, the decontamination works did not allow the reduction of the radioactive Cs below the reference value in the surveyed field of $\sim 6.5 \%$ in Iwate prefecture [13]. These facts imply the presence of Cs adsorption strong enough not to be cleaned by conventional decontamination, which is referred as specific Cs adsorption hereafter.

Our recent studies for the layered clay minerals by positronium (Ps) lifetime spectroscopy coupled with molecular dynamics (MD) simulation revealed the local molecular structures, in which one- and two-clay nanosheets are inserted into the interlayer spaces forming open naospaces [14]. These molecular sites have not been identified by other microscopic techniques, as e.g., transmission electron microscopy and x-ray diffraction, thus having been unconsidered for the molecular model of inorganic layered materials so far. Aside from that, we have developed analytical technique employing element-specific Ps that probes elements located on the surface of open nanospaces [15-18]. This technique is particularly sensitive to Cs as already demonstrated for the analysis of Cs cluster 
on the surfaces of open nanospaces in $\mathrm{SiO}_{2}$ glass [15]. In this study, Cs adsorption on the local molecular sites as stated above is highlighted based on the results of element-specific Ps annihilation spectroscopy. The specific Cs adsorption site is explored to answer the current concern: Why is the radioactive Cs not cleaned up after the decontamination work?

\section{EXPERIMENTS}

Synthetic Na-type saponite $\mathrm{Na}_{0.66}\left[\mathrm{Mg}_{5.34} \mathrm{Li}_{0.66}\right] \mathrm{Si}_{8} \mathrm{O}_{20}[\mathrm{OH}]_{4}$ produced by Kunimine Industries Co. Ltd., Japan, was employed as Cs-unloaded sample. The particle size is approximately $20 \mathrm{~nm}$. Cs loading was conducted by impregnating the Na-type saponite with 1 $\mathrm{M}$ aqueous solution of $\mathrm{CsCl}$. All the samples were initially treated at $423 \mathrm{~K}$ for $24 \mathrm{~h}$ under the vacuum condition of $\sim 10^{-5}$ Torr, which are referred as starting samples.

The sizes of open nanospaces and their fractions were investigated by Ps annihilation lifetime spectroscopy. A fraction of energetic positrons injected into samples forms the bound state with an electron, Ps. Singlet para-Ps ( $p$-Ps) with the spins of the positron and electron antiparallel and triplet ortho-Ps (o-Ps) with parallel spins are formed at a ratio of $1: 3$. Hence, three states of positrons: $p$-Ps, $o$-Ps, and free positrons exist in samples. The annihilation of $p$-Ps results in the emission of two $\gamma$-ray photons of $511 \mathrm{keV}$ with lifetime 125 ps. Free positrons are trapped by negatively charged parts, such as polar elements, and annihilated into two photons with lifetime $\sim 450 \mathrm{ps}$. The positron in $o$-Ps undergoes two-photon annihilation with one of the electrons bound to surrounding molecules with a lifetime of a few ns after localization in angstrom-scale pores. The last process is known as $o$-Ps pick-off annihilation and provides information on the open nanospace size $R$ through its lifetime $\tau_{o-\mathrm{Ps}}$ based on the Tao-Eldrup model [19-20]:

$$
\tau_{o-P s}=0.5\left[1-\frac{R}{R_{0}}+\frac{1}{2 \pi} \sin \left(\frac{2 \pi R}{R_{0}}\right)\right]^{-1}
$$

where $R_{0}=R+\Delta R$, and $\Delta R=0.166 \mathrm{~nm}$ is the thickness of homogeneous electron layer in which the positron in $o$-Ps annihilates. The positron source $\left({ }^{22} \mathrm{Na}\right)$, sealed in a thin foil of Kapton, was mounted in a sample-source-sample sandwich. The starting samples (dehydrated samples) were exposed to the humidity of $\sim 35 \%$ at ambient temperature, where Ps lifetime measurements were performed every 45 min during hydration. Positron lifetime spectra were numerically analyzed using the POSITRONFIT code [21].

The surfaces of open nanospace were investigated by means of momentum distributions of $o$-Ps pick-off annihilation. The momentum spectroscopy is based on the principle that if the positron-electron annihilation accompanies a longitudinal momentum $p$, the resulting annihilation $\gamma$ rays are Doppler shifted from $m_{0} c^{2}$ by $\pm c p / 2$. Here, $m_{0}$ and $c$ are the electron rest mass and velocity of light, respectively. Measurements of the Doppler shifts by $\gamma$-ray energy spectroscopy with high purity Ge detector make it possible to obtain information on the momentum distribution of the positron-electron annihilation pairs. The momentum distribution of $o$-Ps is broadened centered at $511 \mathrm{keV}$ because the pick-off annihilation is influenced by electrons bound to the surrounding molecules [22]-[23]. Thus, the momentum distribution of $o$-Ps pick-off annihilation provides the information on electronic states of pore surfaces. The momentum distribution of $o$-Ps pick-off annihilation was extracted by time-resolved momentum measurements of positron-electron annihilation photons using the recently developed positron-age-momentum correlation (AMOC) spectroscopy [15]. Defining the ratio of the central area over $(-3.6$ to +3.6$) \times 10^{-3} m_{0} c$ to the total area of the momentum spectrum, $o$-Ps pick-off annihilation was parameterized [24]. This is called a pore-surface parameter that was recently found to be sensitive to elements on the surface of open space, as, e.g., Cs [15]-[17]. To investigate specific Cs adsorption, AMOC spectroscopy was conducted for the Cs-loaded sample before and after washing by the hydrochloric acid solution of $\mathrm{pH} 1.0$ several times, which is employed to decontaminate the radioactive Cs from soil [25].

\section{RESUlts AND DisCUSSION}

Ps lifetime spectroscopy for both the Cs-unloaded and loaded samples yields four components of lifetime, where the longest and the second longest components with their relative intensities were attributed to pick-off annihilation of $o$-Ps localized in the open nanospaces denoted as A and B. In Fig. 2 , the sizes $R_{\mathrm{A}}$ and $R_{\mathrm{B}}$ evaluated through above the lifetimes based on Eq. (1) are presented together with their relative intensities, in this work called fractions $f_{\mathrm{A}}$ and $f_{\mathrm{B}}$, as a function of exposure time. It is well seen that the variations of open spaces with exposure time make responses upon Cs loading. The open space size $R_{\mathrm{A}}$ is consistently $\sim 0.3 \mathrm{~nm}$ without any significant change with exposure time for both the Cs-unloaded and loaded samples. The Cs-unloaded sample exhibits decrease in the open space size $R_{\mathrm{B}}$ from $\sim 0.9$ $\mathrm{nm}$ to $\sim 0.6 \mathrm{~nm}$ along with exposure time, whereas the size $R_{\mathrm{B}}$ for the Cs-loaded sample decreases down to $\sim 0.7 \mathrm{~nm}$ more slowly. The fraction $f_{\mathrm{A}}$ for the Cs-unloaded sample increases from $\sim 5 \%$ to $\sim 15 \%$ with exposure time in contrast to the decrease of the fraction $f_{\mathrm{B}}$ from $\sim 10 \%$ to $\sim 2 \%$, which are synchronized with the variation of $R_{\mathrm{B}}$ in the time scale of $\sim 10$ days. The Cs-loaded sample exhibits the increase in the fraction $f_{\mathrm{A}}$ in a similar manner to that of Cs-unloaded one but in much longer time scale of $\sim 19$ days. The fraction $f_{\mathrm{B}}$ initially exhibiting high value of $\sim 20 \%$ decreases down to $\sim$ $3 \%$ synchronizing with the increase of $f_{\mathrm{A}}$.

Generally, the interlayer spaces expand together with hydration. It is thus unlikely that the open space A with the constant size of $\sim 0.3 \mathrm{~nm}$ corresponds to the interlayer spaces. The open space $B$ with its size ranging from $\sim 0.6$ to $\sim 1 \mathrm{~nm}$ is in turn too large to relate with the interlayer spaces. We thus introduced two kinds of local molecular structures simulated by molecular dynamics (MD) calculations to explain the long-term variation of open nanospaces observed for the Cs-unloaded saponite in Fig. 2 [14]. One of the molecular structures is called type A, in which a one-clay nanosheet is inserted into the interlayer spaces forming the open space with the size of $\sim 0.3 \mathrm{~nm}$ (see inset in Fig. 2 (a)). Another is 
type B, in which a two-clay nanosheet is inserted into the interlayer spaces forming the large open space with the size of $\sim 0.9 \mathrm{~nm}$ (see inset in Fig. 2 (c)). Note that the sizes of open spaces simulated by MD calculations are in agreement with those observed by Ps annihilation spectroscopy. In a dehydrated state, the local molecular structure of type B dominantly exists as deduced from the higher fraction of $f_{\mathrm{B}}$. $\mathrm{H}_{2} \mathrm{O}$ molecules adsorbed at $\mathrm{Na}^{+}$cations in the interlayer spaces due to hydration trigger off the onset of rheological motion of nanosheets in parallel to the layer direction. One of two nanosheets inserted into the interlayer space of type B is thus released away, with the type A with smaller open spaces getting to dominant for a hydrated state. The open space size $R_{\mathrm{B}}$ thus decreases with increasing exposure time, though the open space size $R_{\mathrm{A}}$ remains constant. Correspondingly, the fraction $f_{\mathrm{B}}$ decreases and the fraction $f_{\mathrm{A}}$ oppositely increases with exposure time.

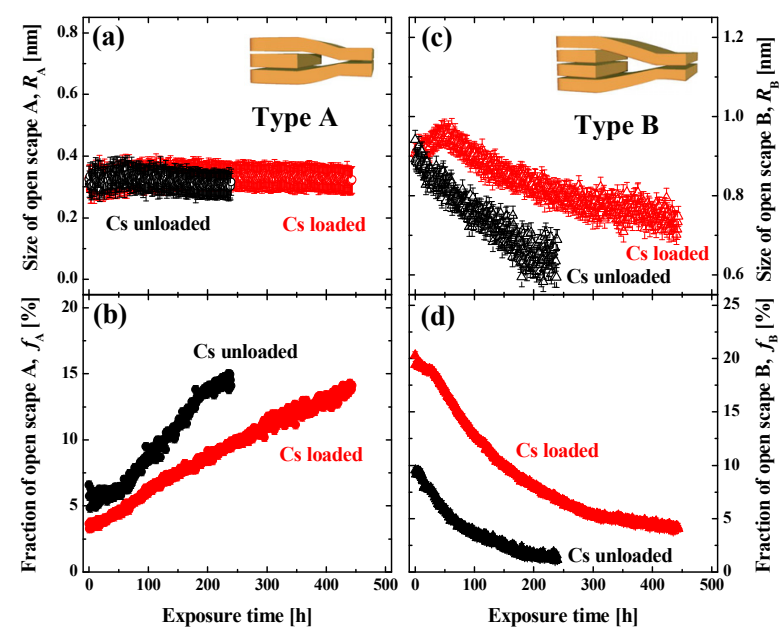

Fig. 2. Sizes of open spaces (a) $R_{\mathrm{A}}$ (solid circles) and (c) $R_{\mathrm{B}}$ (solid triangles) with their fractions (b) $f_{\mathrm{A}}$ (open circles) and (d) $f_{\mathrm{B}}$ (open triangles) as a function of exposure time obtained for the Cs-loaded saponite sample. The data for the Cs-unloaded sample taken from our literature (14) is added for comparison. Types A and B are schematically illustrated in (a) and (b), respectively.

Basically, the variations of open spaces observed for the Cs-loaded saponite can be also explained by the above mentioned water-induced structural modification of types A and $\mathrm{B}$, which are dominantly present in hydrated and dehydrated states, respectively. Upon Cs loading, the $\mathrm{Na}^{+}$ cations in the interlayer spaces are ion-exchanged by $\mathrm{Cs}^{+}$that is less attractive to $\mathrm{H}_{2} \mathrm{O}$ molecules due to its lower hydration degree. One of the two nanosheets is sluggishly released away from the molecular structure of type B with less $\mathrm{H}_{2} \mathrm{O}$ molecules getting toward to type A. The fraction $f_{\mathrm{A}}$ for the Cs-loaded saponite thus increases more slowly with exposure time than that of Cs-unloaded one together with corresponding decreases of $f_{\mathrm{B}}$ as well as $R_{\mathrm{B}}$, though the size of small open space $R_{\mathrm{A}}$ is unchanged.

Fig. 3 shows the positron-age dependent pore surface parameters obtained for the hydrated and dehydrated samples, which probe the elemental environment around open spaces $\mathrm{A}$ and $\mathrm{B}$ in the molecular structures of types $\mathrm{A}$ and $\mathrm{B}$, respectively. The pore surface parameters of open space $A$ for the Cs-loaded saponite are significantly higher than those of the Cs-unloaded one in the older positron age region. As is already demonstrated, the pore surface parameter in the older positron age region is highly specific to elements located on the surfaces of open nanospaces, since the process of $o$-Ps pick-off annihilation is influenced by their electron momenta [15-18]. The enhancement of the pore surface parameters upon Cs loading is typical for $o$-Ps pick-off annihilation with the electrons bound to Cs [15]. It is thus concluded that Cs locally adsorbs on the surface of open space A in the molecular structure of type A, as illustrated in the inset of Fig. 2 (a). The pore surface parameters of open space B in the molecular structure of type B are also enhanced upon Cs loading, indicating adsorption of more $\mathrm{Cs}$ on the surface of open space B, as illustrated in the inset of Fig. 2 (b).
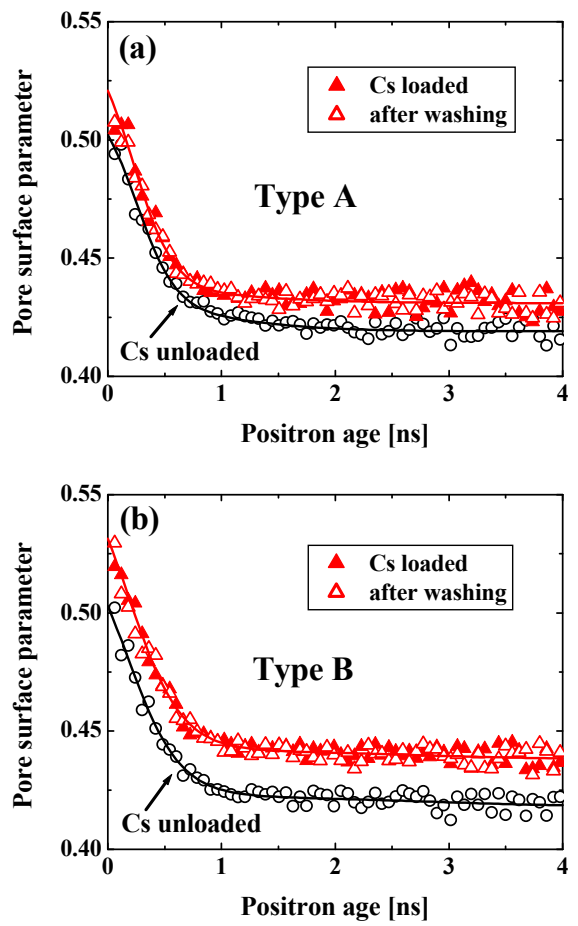

Fig. 3. Positron-age dependent pore surface parameters for (a) hydrated and (b) dehydrated samples measured by AMOC spectroscopy. The data for Cs-unloaded, Cs-loaded, and Cs-loaded and then acid-washed saponite are indicated with open circles, solid triangles, and open triangles in both (a) hydrated and (b) dehydrated saponite. The solid lines are drawn for guiding the eye. Note that the types A and B dominantly exist in (a) hydrated and (b) dehydrated saponite, respectively. The data shown in (a) and (b) are thus exclusively resultant from the elemental environment of open spaces A and B, respectively.

It is now recognized that $\mathrm{Cs}$ adsorbs on the surfaces of the open nanospaces A and B in the local molecular structures of types A and B revealed by a series of Ps annihilation spectroscopy. Our next interest is whether the open spaces A and $\mathrm{B}$ act as specific adsorption site for Cs. To confirm it, the elemental analysis of open spaces A and B by AMOC spectroscopy was again conducted for the Cs-loaded sample, which was washed by the hydrochloric acid solution of $\mathrm{pH}$ 1.0 strong enough to decontaminate the radioactive Cs [25]. Noticeable here is that the pore surface parameters are unchanged before and after washing by hydrochloric acid solution for both the cases of open spaces A and B (see Fig. 3 ). This evidences that $\mathrm{Cs}$ adsorbed on the surfaces of both open spaces $\mathrm{A}$ and $\mathrm{B}$ cannot be removed even by the strong acid, the open spaces A and B thus acting as specific Cs 
adsorption sites. It should be noted here that there exist the clay-nanosheet edge sites in both the open spaces A and B. The clay-nanosheet edge sites are expected to be chemically active for molecular adsorption as those of cup-stacked carbon nanotube. Furthermore, the wedge-shaped part similar to the frayed-edge site generally appeared after long-term pedogenetic weathering is already available both in the open spaces A and B. The frayed-edge site is known to capture Cs with much greater affinity than the expanded layers [7,26]. It is reasonably inferred that such local molecular sites characteristic for the open spaces A and B are responsible for the heavily adhesive $\mathrm{Cs}$ adsorption observed here.

Based on the present findings by element-specific Ps annihilation spectroscopy for the Cs-unloaded and Cs-loaded saponite samples, the following adsorption behavior is presumed for the radioactive $\mathrm{Cs}$ in soil. As is generally accepted, the radioactive Cs selectively adsorbs on the surface of interlayer spaces in clay minerals. Such physisorbed $\mathrm{Cs}$ on the clay surface could be removed by the conventional decontamination work as, e.g., high-pressure water jet cleaning. In addition, a population of Cs adsorbs on the surfaces of both open spaces A and B formed by inserting one- and two-clay nanosheets into the interlayer spaces, respectively. The one- and two-nanosheet inserted local molecular structures of types A and B dominantly exist in hydrated and dehydrated states, respectively, thus implying that both the molecular structures favorably appear under a wide variety of hydration conditions in soil. Cs adsorbed on the surfaces of both the open spaces A and B is so adhesive that cannot be removed even by the hydrochloric acid solution of $\mathrm{pH}$ 1.0. There exists characteristic local molecular site as a wedge-shaped part both in the open spaces A and B, where the hexagonal cavities similar to the size of $\mathrm{Cs}^{+}$cations are faced each other. It is expected that this molecular site strongly captures $\mathrm{Cs}^{+}$cations, being responsible for the specific Cs adsorption. In addition to that, the open space B possesses the chemically-active molecular site as a clay-nanosheet edge. The open spaces A and B thus act as the specific Cs adsorption site, where conventional decontamination could not be applied. Radioactive ${ }^{137} \mathrm{Cs}$ that still appears beyond the reference value after the decontamination work would originate from the specific Cs adsorption clarified in the present work.

\section{CONCLUSION}

To gain an insight into current unsuccessful decontamination of radioactive ${ }^{137} \mathrm{Cs}$ from soil, the adsorption sites of heavily adhesive Cs were investigated for the layered clay mineral by means of element-specific Ps annihilation spectroscopy. Beside the interlayer $\mathrm{Cs}^{+}$cations, a population of $\mathrm{Cs}$ is able to adsorb on the surfaces of open nanospaces $A$ and $B$ with their sizes of $\sim 0.3 \mathrm{~nm}$ and $\sim 0.9 \mathrm{~nm}$, which are formed by inserting one- and two-clay nanosheets into the interlayer spaces. The open space B is found to be more susceptible to Cs adsorption than in the open space A owing to its larger size. Cs adsorbs on the surfaces of both the open nanospaces $\mathrm{A}$ and $\mathrm{B}$ is so adhesive that cannot be removed even by the hydrochloric acid solution of $\mathrm{pH} 1.0$, the open nanospaces A and B thus acting as the specific Cs adsorption site. The characteristic local molecular sites as a clay-nanosheet edge and a wedge-shaped frayed part available in the open spaces A and B could be responsible for the specific Cs adsorption. Such heavily adhesive Cs could not be removed by the conventional decontamination work. The local molecular structures of types A and B dominantly exist in hydrated and dehydrated states, respectively, thus implying that both the molecular structures favorably appear under a wide variety of hydration condition in soil. The present findings unambiguously provide the molecular evidence of specific Cs adsorption and are thus of importance for further sophisticated decontamination of the radioactive Cs from the soil environment.

\section{ACKNOWLEDGMENT}

The authors are indebted to K. Fujimoto (Tokyo Gakugei University), M. Nakata (Tokyo Gakugei University), N. Shikazono (Keio Univeristy), K. Kawamura (Okayama University), Y. Kobayashi (National Institute of Advanced Industrial Science and Technology), K. Ito (National Institute of Advanced Industrial Science and Technology), and M. Kamaya (Kogakuin University) for fruitful discussion.

\section{REFERENCES}

[1] R. Weiss and J. Bourgeois, "Understanding Sediments-Reducing Tsunami Risk," Science, vol. 336, pp. 1117-1118, 2012.

[2] P. C. Burns, R. C. Ewing and A. Navrotsky, "Nuclear fuel in a reactor accident," Science, vol. 335, pp. 1184-1188, 2012.

[3] N. Kinoshita, K. Sueki, K. Sasa, J. Kitagawa, S. Ikarashi and T. Nishimura, Y. Wong, Y. Satou, K. Handa, T. Takahashi, M. Sato, T. Yamagata, "Assessment of individual radionuclide distributions from the Fukushima nuclear accident covering central-east Japan," in Proc. Natl. Acad. Sci. U.S.A., vol. 108, pp. 19526-19529, 2011.

[4] T. J. Yasunari, A. Stohl, R. S. Hayano, J. F. Burkhart, S. Eckhardt, and T. Yasunari, "Cesium-137 deposition and contamination of Japanese soils due to the Fukushima nuclear accident," in Proc. Natl. Acad. Sci. U.S.A., vol. 108, pp. 19530-19534, 2011.

[5] N. Yoshida and J. Kanda, "Tracking the Fukushima Radionuclides," Science, vol. 336, pp. 1115-1116, 2012.

[6] A. Cremers, A. Elsen and P. De Preter, A. Maes, "Quantitative analysis of radiocaesium retention in soils," Nature, vol. 335, pp. 247-249, 1988 .

[7] B. C. Bostick, M. A. Vairavamurthy, K. G. Karthikeyan, and J. Chorover, "Cesium adsorption on clay minerals: an EXAFS spectroscopic investigation," Environ. Sci. Technol., vol. 36, pp. 12670-12676, 2002.

[8] Y. Morino, T. Ohara, and M. Nishizawa, "Atmospheric behavior, deposition, and budget of radioactive materials from the Fukushima Daiichi nuclear power plant in March 2011," Geophys. Res. Lett., vol. 38, pp. L00G11, 2011.

[9] Y. Kim and R. J. Kirkpatrick, "23Na and 133Cs NMR study of cation adsorption on mineral surfaces: Local environments, dynamics, and effects of mixed cations," Geochem. Cosmochim. Acta, vol. 61, pp. 5199-5208, 1997.

[10] Japanese Ministry of the Environment. Decontamination Roadmap for the Special Decontamination Areas. (2012). [Online]. Available: http://josen.env.go.jp/en/roadmap/

[11] Ministry of Education, Culture, Sports, Science, and Technology, Japan. Results of nuclide analysis of gamma-emitting nuclides (Cs-134, Cs-137 and Ag-110m) (Second Distribution Survey) (March 1, 2012). [Online]. http://radioactivity.mext.go.jp/en/contents/6000/5635/view.html, 2012.

[12] Japan Atomic Energy Agency. (2012). Report of the Results of the Decontamination Model Projects. [Online]. Available: http://www.jaea.go.jp/fukushima/decon04/decon04-ke_e.html

[13] Iwate Prefecture Web Site. (2012). [Online]. Available: http://www.pref.iwate.jp (in Japanese) 
[14] K. Sato, K. Fujimoto, K. Kawamura, and W. Dai, M. Hunger, "Rheological Mechanism of Long-Term Self-Assembly in Saponite Nanoparticles," J. Phys. Chem. C, vol. 116, pp. 22954-22959, 2012.

[15] K. Sato, H. Murakami, K. Ito, K. Hirata, and Y. Kobayashi, "Probing the Elemental Environment around the Free Volume in Polymers with Positron Annihilation Age-Momentum Correlation Spectroscopy," Macromolecules, vol. 42, pp. 4853-4857, 2009.

[16] K. Sato, K. Fujimoto, M. Nakata, and T. Hatta, "Diffusion-Reaction of Water Molecules in Angstrom Pores as Basic Mechanism of Biogenic Quartz Formation," J. Phys. Chem. C, vol. 115, pp. 18131-18135, 2011.

[17] K. Sato, "Origin of Organism-Dependent Biogenic Silica Quartz Formation," J. Phys. Chem. B, vol. 115, pp. 14874-14877, 2011.

[18] K. Sato, W. Sprengel, "Element-specific study of local segmental dynamics of polyethylene terephthalate upon physical aging," J. Chem. Phys., vol. 137, pp. 1049061-1049067, 2012.

[19] S. J. Tao, "Positronium annihilation in molecular substances," J. Chem. Phys., vol. 56, pp. 5499-5510, 1972.

[20] M. Eldrup, D. Lightbody, and J. N. Sherwood, "The temperature dependence of positron lifetimes in solid pivalic acid," Chem. Phys. vol. 63 , pp. 51-58, 1981.

[21] P. Kirkegaard, and M. Eldrup, "Positronfit extended: A new version of a program for analysing position lifetime spectra," Comput. Phys. Commun., vol. 7, pp. 401-409, 1974.
[22] K. Sato, K. Ito, K. Hirata, R. S. Yu, and Y. Kobayashi, "Intrinsic momentum distributions of positron and positronium annihilation in polymers," Phys. Rev. B, vol. 71, pp. 0122011-0122014, 2005.

[23] K. Sato, D. Shanai, Y. Hotani, T. Ougizawa, K. Ito, K. Hirata, Y. Kobayashi, "Positronium formed by recombination of positron-Electron pairs in polymers," Phys. Rev. Lett., vol. 96, pp. 2283021-2283024, 2006

[24] K. Sato, F. Baier, W. Sprengel, R. Wurschum, and H.-E. Schaefer, "Study of an order-disorder phase transition on an atomic scale: the example of decagonal Al-Ni-Co quasicrystals," Phys. Rev. Lett., vol. 92, pp. 1274031-1274034, 2004.

[25] Y. Wen, J. Song, W. Liu, and Z. Qin, "Decontamination of radioactive cesium in natural $\mathrm{NaCl}$ by amide-type open-chain crown ethers(II)," $J$. Radioanalytical and Nuclear Chem., vol. 253, pp. 167-170, 2002.

[26] B. L. Sawhney, "Selective sorption and fixation of cations by clay minerals: a review," Clay Clay Minerals, vol. 20, pp. 93-100, 1972.

Kiminori Sato received the Ph. D from Department of Applied Physics, the University of Tokyo, Japan, in 2001, K. Sato has concentrated on researches on complex phenomena originated from angstrom- and nano-scale open spaces in material as well as in geological environment. 